\title{
Q9intish
}

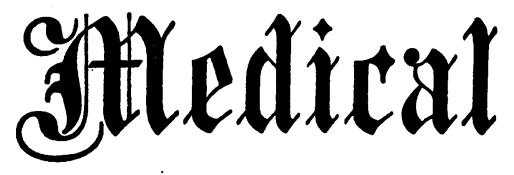

dֶourmal.

THE JOURNAL OF THE BRITISH MEDICAL ASSOCIATION.

LONDON： SATURDAY, JULY 6TH, 1912.

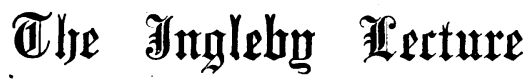 \\ ON \\ PUERPERAL INFECTION, WITH SPECIAL REFERENCE TO VACCINE TREATMENT:* \\ BY}

J. FURNEAUX JORDAN, F.R.C.S., SERGEON TO THE BIRMINGHAM HOSPITAL FOR WOMEN AND THE MATERNITY HOSPITAL.

IT is impossible to cover the whole of the subject of puerperal fever. I propose, therefore, to refer to the incidence of the disease, to some of its types which are fairly distinct, to the cause or causes as far as we know them, to some of the pathological changes, and the treatment, preventive and curative.

Until quite recently there has been great difficulty here in the way of making any study of these cases, because there has been little or no institutional treatment for them. They were, and are, certainly not wanted at the Maternity Hospital. No operating surgeon has ever been anxious to mix these cases with his operation cases; but some time ago now the Women's Hospital at Sparkhill had a ward specially built for the reception of septic cases, and into this we are able to take cases of puerperal fever. We also have an arrangement with the Health Committee of the City Council by which those cases of puerperal fever reported to the medical officer of health which are unable to get any proper attention at home are sent in by him to the Women's Hospital. Into this ward last year 36 cases of puerperal fever were admitted. Cases of some septic condition or other - for example, a parametritis or a pyosalpinx, ultimately following on and due to the confinement -are not included in these 36 . They were cases of puerperal fever coming on within a few days of the confinement. In the first three months of this year another 9 cases have been admitted; of these 45 cases 15 have died, 6 of the 15 within three days after admission, 6 on days between the seventh and fourteenth after admission, and 3 on the forty-fifth, fifty-ninth, and fifty - ifth day respectively. Even a preliminary examination of the cases shows that the first 6 were cases far too far gone at the time of admission for any treatment to have a chance of success; the second 6 were severe cases in which treatment was tried with very little effect; while the 3 who survived for so long were at death's door many times before they finally succumbed.

I propose to dwell for a short time upon three types of case : (1) Those that are of a mild type and recover easily. (2) Those that are acutely infected and die quickly. (3) Those that are severely infected or ill for a long time, and usually, but not always, recover.

\section{Mild Type.}

In considering the first class-the less severely infected or the mild type-the most noticeable feature about them is that the temperature fell to normal in all but one of them within forty-eight hours after admission; in the one exception the fall was a gradual one, reaching normal on the fourth day. This is really a curious fact when one considers the following information about them. There were 10 cases of this type; we will call the day of confinement in all of them the first day:

1. Fever started on the seventh day, admitted to hospital on the eleventh day with a temperature of $99.6^{\circ}$.

2. Fever started on the third day, admitted to hospital on the twenty-first day with a temperature of $102.6^{\circ}$

3. Fever started on the fifth day, admitted to hospital on the seventh day with a temperature of $101^{\circ}$.

4. Fever started on the second day, admitted to hospital on the seventh day with a temperature of $100.8^{\circ}$.

5. Fever started on the second day, admitted to hospital on the fourth day with a temperature of $100^{\circ}$.

6. Fever started on the third day, admitted to hospital on the twelfth day with a temperature of $100.6^{\circ}$.

7. Fever started on the twelfth day, admitted to hospital on the seventeenth day with a temperature of $99.8^{\circ}$.

8. Fever started on the third day, admitted to hospital on Fever started on the third day, admitted to $h=$

9. Fever started on the twelfth day, admitted to hospital on the nineteenth day with a temperature of $103.8^{\circ}$.

10. Fever started on the third day, admitted to hospital on the tenth day with a temperature of $99.5^{\circ}$.

The duration of the fever, therefore, extended from two to eighteen days before admission, yet after admission the temperature fell to normal in all of them in practically the same time-namely, about two days. It looks as though in each case the temperature was only waiting for treatment to come down to normal. The symptoms on admis. sion were usually the rise of temperature already men. tioned, quickening of the pulse to 100 or up to 120 , but not higher ; furred tongue; uterus big and tender; lochia usually profuse and foul smelling; constipation; and a general appearance of being ill. Three of the patients had a blunt curetting done the morning after admission: in two of the three some septic retained placenta was found; in the third case the uterus was clean. On admission to hospital the change from a poor, dirty little house, from inefficient attendance, from improper feeding, and in some cases from actual neglect, to a comfortable, warm, clean bed, and to good nursing and feeding; the change from the constant worry and anxiety at home to the general sense of well-being and comfort in the hospital; the change from constipation to a thorough evacuation of the bowels : the change from dirt to absolute cleanliness, helped usually by a gentle antiseptic vaginal douche, and a tonic such as mist. ferri, seems to have been sufficient for a rapid recovery in these cases. We can only assume that the infection is slight, in some cases purely local, and that the resisting powers of the patient only want a little help to bring about a recovery.

\section{Acute Infection: Early Death.}

Early onset of the symptoms, a rise of temperature to $103^{\circ}-105^{\circ}$, profuse sweats, a pulse of 120 and rising as high as 160 , are sufficient to cause grave anxiety. In some of these cases the patient, however, looks fairly well, and except for feeling hot she may be quite comfortable; the pulse has a good volume, the bowels may be acting (but this usually only from treatment), the abdomen is slightly distended but quite soft, palpable, and free from tenderness; there is no uterine tenderness, there is nothing abnormal to be felt in the pelvis, and one may

[2688] 
be inclined to give a hopeful prognosis. In reality the prognosis is very grave: the pulse gets weaker and weaker, the tongue becomes furred, and dry sordes appear on the lips and gums; the nourishment taken becomes less and less, rigors may or may not occur, prostration becomes more marked, the distension of the abdomen increases, constipation gives way to septic diarrhoea with involuntary evacuation, and the patient rapidly sinks from acute toxin poisoning. The rapidly fatal cases at Sparkhill were all in the latest stages of acute infection on admission, and it was impossible to render them immune to the infection; but that these cases are not necessarily fatal, and that they are not naturally bound to follow the distressing course I have briefly outlined, is known to all and is well illustrated by three cases I -have seen recently outside the hospital: The history of these cases is as follows:

\section{Case I.}

Mrs. F., patient of $\mathrm{D}_{i}$. Middleton. Confinement. Confined on November 28th, 1911; normal; baby weighed $6 \mathrm{lb}$. $15 \mathrm{oz}$ Temperature rose to $103^{\circ}$ on the third day; the uterus was washed out with an antiseptic douche, a turpentine enema was given, and the temperature fell to $101^{\circ}$. Vasinal douches were subsequently given, the bowels were kept open, and tonics were administered. The patient felt better, and for four days the temperature hovered between $99.6^{\circ}$ and $101.5^{\circ}$. On the eighth day she had a rigor, the temperature rose to $105^{\circ}$, and she felt very ill. I saw her with Dr. Middleton, and, under an anaesthetic, I took two swabs from the interior of the uterus for subsequent bacterial investigation, and also by exploration cusequention satisfed myself that the interior of the uterus did not contain the next two days to normal, only to rise on the third day after the nigor to $103^{\circ}$. In the meantime Dr. Mackey had examined the rigor to $103^{\circ}$. In the meantime Dr. Mackey had examined of streptococcus. Of the vaccine he prepared from this 5 minims (containing nearly 30 million streptococci) were and never rose again. A second injection of 35 million was given four days later.

Case II.

Mrs. H., patient of Dr. L'Estrange Burges. Normal confinement on February 29th, 1912; the temperature normal, and patient going on well till the seventh day, when the tempera ture rose to $104^{\circ}$. I saw her with Dr. Burges, and carried out the same procedure as in Case $I$, but, in addition, injected her with 4 minims of the stock vaccine of Case I- that is, with 25 million streptococci; her temperature fell to $101^{\circ}$ in the nex four hours, but hovered between $101^{\circ}$ and $102^{\circ}$ for the next two days, and then rose to $103^{\circ}$, when she had an injection of 25 million streptococci in 5 minims of her own vaccine prepared by Dr. Mackey from the swabs I had taken. Her temperature came down again to $101^{\circ}$ for two days, and then rose to $104^{\circ}$, when she had 50 million of streptococci in 8 minims of her own vaccine injected. In less than twentyfour hours the temperature fell from $104^{\circ}$ to normal and never rose again.

Case III.

Mrs. F., patient of Dr. Cochrane; second child, normal confinement. Three days later the temperature rose to $104.2^{\circ}$ with pulse of 144 . When I saw her she presented the usual signs of an acute infection in : the early stages. Since the patient was hardly able to afford the preparation of her own vaccine, a stock vaccine of the streptococcus prepared by $\mathrm{Dr}$. Mackey from a previous case.was used, 25,30 , and 35 million streptococci being injected every third day. After the second injection her temperature came down and never again gave any cause for anxiety.

These cases when first seen, especially Case II, resembled in every way the cases which at a later stage have been admitted to Sparkhill only to die in a very short time. In these private cases treatment by vaccines, etc., was begun early in the disease, whereas in the six cases at Sparkhill that died within two or three days after admission they were only admitted to the hospital on the third, fourth, fourth, tenth, sixth, and fifth days respectively of their illness. In addition to the vaccine treatment in the three cases outlined; attention was paid to the bowels, suitable diet was administered, tonics given, and all of them had the benefit of good nursing. 'It is; of course, im vossible to say for certain that, with the ordinary measures of treatmient and without the vaccine, they wouli have developed the later symptoms such as those of the rapidly fatal cases at Sparklill, but one cannot overlook the sact that in each of them a rapid and permanent recovery from a very serrious condition followed this treatment. Before leaving these acutely infected cases, I must refer briefly to the pathological changes found post mortem.
Case IV.

Mrs. L., aged 28 ; five previous preonancies; no miscarriages. Admitted to Sparkhill on May 5th, 1911, with a temperature of $105^{\circ}$, pulse $130^{2}$. Delivered eleven days previously by a midwife. Two days after delivery she had an attack of acute wife. Two days after delivery she had an attack of acute abdominal pain for which the doctor was sent for; vaginal
douches were given and medicine. On the next day she had three rigors. The pain and the rigors continued until her admission to the hospital on the twelfth day, when she was acutely septic and very ill; a most offensive vaginal discharge; no definite abdominal symptons. On May 7th, two days after admission, Dr. Edge took swabs from her uterus and dicl a blunt curetting. On the next day her temperature was $101.6^{\circ}$ in the morning, but in the evening she had a rigor, and her temperature rose to $105.6^{\circ}$; she had an injection of stock vaccine (25 million streptococei), and her temperature fell to $103^{\circ}$ till the next evening, when it rose again to $105^{\circ}$. A second injeciion of stock vaccine was given, this time 30 million streptococci ; her temperature came down for a few hours to $100^{\circ}$, but rose on the next evening to $105.4^{\circ}$, when she died. Dr. Mackey's report on the swabs showed an infection of streptoMackey's report on the swabs showed an infection of streptothe patient was near the end when it was ready it was never injected.

Post-inortem Examination.-Body emaciated, no general peritonitis, but some puralent fluid in the pelvis; uterus large and flabby, like an empty bladder, and containing some old blood clot, but not offensive nor obviously septic; the Fallopian tubes contained pus, the left one being distended; the pus escaping from the fimbriated end when the tube was handled; no thrombosis in the pelvic veins; kidneys large and pale; spleen large, about $10 \mathrm{oz}$; liver honeycombed with gas bladlers, and the wall of the intestine emphysematous in parts, evidently from a gas-producing bacillus; lungs oedematous; heart extremely flabby, no endocarditis.

\section{CASE v.}

Mrs. H. Admitted to the Women's Hospital under Mr. Martin on February 19th. Delivered eight days previously by a midwife, who examined the patient several times before delivery. Placenta expressed without any difficulty. On the second day the patient felt very ill and feverish, having a rigor which lasted a long time, and two days later a doctor was called in who curetted the uterus. She, however, continued feverish and shivering until the eighth day, when she was admitted with a temperature of $104.8^{\circ}$, pulse 140 , tongue dry and furred, abdomen tender and distended, uterus tender and somewhat fixed by a tender swelling to the right of the cervix. The interior of the uterus was bluntly curetted without an anaesthetic, and swabbed with pure carbolic, and left with an iodoform gauze drain. Swabs of the uterus were tuken before rhe gauze drain. Swabs of the uterus were taken before she was curetted, and report showed infection by streptococcus, but a streptococcus not the same as that found in other puerperal cases. A vaccine was prepared, but the patient clied before it was ready for injection, her tem
day after admission to $105.4^{\circ}$, when she died.

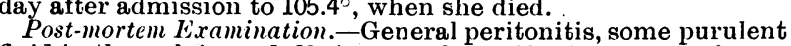
fluid in the pelvis, and fibrino-purulent adhesions everywhere; fluid in the pelvis, and fibrino-purulent adhesions every where; clots and (?) placenta; the broad ligaments infiltrated, and on cross section honeycombed with pus; the Fallopian tubes, though not much dilated, contain thick yellow pus; the kidneys are congested, their pelves dilated, and they look moderately fatty; liver large, and fatty degeneration; spleen large and soft; lungs congested; heart dilated, no endocarditis.

Case vi.

Mrs. E., aged 22; married sixteen months; confined on December 18th ; normal confinement. On Christmas day acute pain in the abdomen, and on the next day diarrhoea and sickness which persisted profusely for twenty-four hours. When seen by Mr. Hewetson on December 27 th was very ill and acutely septic, with a pulse of 140 ; she was given a morphine suppository which stopped the diarrhoea, and was admitted to the Women's Hospital on December 29th. Great pain in the abdomen, chietly just below the costal margins when taking a deep breath; the sickness was better, there was albuminuria, but no pus in the urine; she was very emaciated, suffering countenance tongue dry in the middle heart and lun, suffering coundistension of the in the middle. heart and lungs normal, some distension of the abdomen with general tenderness, uterus night after admission with fair results. In spite of treatment she gradually got worse, and died on January 3rd.

Post-mortem Examination.-Body pale and thin. Abdomen : General fibrino-purulent peritonitis from the pelvis to the diaphragm; uterus empty and not obviously septic; nothing wrong with the tubes, no purulent pelvic cellulitis; kilneys fair; liver large and fatty degeneration well marked. Thorax : Pericardium contains 6 ir 8 oz. of thick pus; heart flabby, but no endocarditis; a-recent pleurisy over the left lung which is collapsed, no effusion and no abscesses in the lung; right lung adherent in several places, no abscesses, no pleural effusion.

\section{CASE VII.}

Mrs. S., aged 26; fourth confinement on September 3rd last year; normal, attended by a midwife. On the second day after confinement had a rigor, became rapidly ill, and was seen by a dispensary doctor on September 7 th. I saw her with him on the same day. I found her in the midst of the filthiest and
most poverty stricken surroundings, and lying covered where 
the skin was exposed by myriads of flies, of which she took not the slightest notice; pulse 148 , temperature $104^{\circ}$; abdomen distended and tender in the lower part; uterus and broad ligaments very tender; respiration hurried and very difficult. I admitted her to the Women's Hospital at once. She never recovered complete consciousness; her temperature varied from
$103^{\circ}$ t $105.8^{\circ}:$ septic diarrhoea supervened, with rapid prostra. tion and death five days after admission.

Post-mortem Examination.-The abdomen contained a few ounces of fluid, but no pus; intestines and stomach were much distended with gas; uterus pale, rather large and soft, its cavity empty, and not very septic looking; in the wall of the nterus empty, and not very septic looking; in the wall of the uterus
there were one or two small abscesses; from the left brcad ligament pus was obtained by squeezing, and the left tube contained some semipurulent fluid ; the pelvic veins were not tained some semipurulent fluid; the pelvic veins were not
thrombosed; the spleen was large and septic; liver large, very soft and fatty degeneration, but no abscesses were seen; the kidneys were pale, large, cortex swollen and very fatty-
kidners typical of septicaemia. Thorax: In both pleural kidneys typical of septicaemia. Thorax: In both pleural
cavities there were sone ounces of semipurulent fluid; both lunis showed purulent lymph on their exterior, and both on section showed well-marked oedema and septic pneumonia; the substance of the lungs was very friable, and in both there were numerous small abscesses; the pericardium contained a slight excess of semipurulent fluid, and there was early pericarditis noticeable on right auricular appendix; the heart muscle was pale and fatty, and the cavity showed a large amount of septica few recent smoll vegetations, and the aorta some aortitis of the aortic sinus.

Reference has already been made to those cases that, although acutely infected and very ill, were in hospital for a long time, and ended usually in recovery. If the histories of these cases are not becoming wearisome to you, I would like, as briefly as possible, to record some of these.

CASE viri.

Mrs. H., aged 27. Admitted to hospital Norember 15th, baby born on November 9 th; examined once before the membaby ruptured by the nurse attending her, but at the actual birth of the baby no one was present except a neighbour. Dr. Sangster was calied in to remove the piacenta, followerl later by profuse haemorrhage; she had a rigor the same day, but aiter this felt well until the third day. On the first lay she and her bedclothes were wet through by the rain coming in through the roof. On the third day she began to feel hot, and her temperature remained high until she was sent to the Wcmen's Hospital on November $15 t h$ with a temperature of $105^{\circ} ;$ pulse 114 .
On almission, abdomen slightly distended, no tenderness, uterus above the lrim, profuse septic discharge, tongue furred, patient very anaemic. Next day, on the 16th, the temperature came very anaemic.
down to $103^{\circ}$ and her pulse to 118 ; blunt curretting was done, down to $103^{\circ}$ and her pulse to lis; blunt curretting was clone, and some small fragments of membrane got away. Before the curetting was done two sterile swabs were taken of the uterine
discharge from which to grow cultures; these cultures showed streptococci and Bacillus coli. The temperature dial not fall until the third day, when it came down to $101.4^{\circ}$. On the evening of the third day she had an injection of her own vaccine, 4 minims. On the fourth day temperature came down to $100.2^{\circ}$, but on the fifth, sixth, and seventl days rose again to $102^{\circ}, 104^{\circ}$, and $103^{\circ}$. On the seventh day she had another injection of vaccine, 5 minims. Between then and the thirteenth day her temperature was down in the morning to $99.2^{\circ}$ and twice to normal, but up in the evening to $102^{\circ}$, and on the twelfth day to $103^{\circ}$. On the thirteenth day another injection of vaccine, 6 minims, was given, after which her temperature hovered above normal day after day for a fortnight. She had two more injecnormal day after day for a fortnight. She had two more injecthe temperature was higher in the evening than the previous the temperature was higher in the evening than the previous
fortnight; though down to normal in the mornings, and it confortnight; though down to normal in the mornings, and it conDuring all this time the patient complained of pain in the wrist, pain in the legs, and especially in the left ankle. The joints were swollen slightly, acutely tender, but as time went on both the swelling and the tenderness gradually disappeared, except in the left ankle, where a stwelling persisted and the tenderness remained acute. Repeated examination of the chest. of the abdomen, and the pelvis failed to elicit any cause for her temperature-the serous effusion round the ankle-joint not being sufficient to account for such an irregular temperature. On the fiftieth day a slight tenderness and swelling was noticed over the back of the sacrum, and on the next day the swelling had increased considerably; an incision was made into it, and a large quantity of pus let out. Her temperature fell the next day to normal.

Her recovery from this time onwards, though very slou, was steady. Massage to the left leg and ankle helped her to steady. Massage to the left leg and ankle helped her to
gradually recover the use of the leg, and for a week before she gradually recover the use of the leg, and for a week before she
was discharged to the convalescent home she was able, with wome assistance, to put her foot to the ground and hobble abort

This, with the case of Mrs. D. (Case $\mathrm{x}$ ), are both examples of infection in which quite late in the course of the disease a local abscess forms, the opening of which scems to put an end to the invasion of the tissues by the germs, and to allow of the immunity which they gradually acquire to take effect.
CASE IX.

Mrs. T., aged 28. Delivered at her home on October 31st, at 10 a.m. Fifth pregnancy. The nurse arrived at $8.30 ; \mathrm{mem}$ branes ruptured 9.15; some difficulty in delivery from the size of the head and shoulders. Baby was asphyxiated, and while it was being attended to the placenta was expelled, not intact. wfter the third stage rather more than the normal loss. On After the third stage rather more than the normal loss. On
November 2 nd temperature was $101^{\circ}$ and pulse 120 when the nurse visited her; the bowels had not been moved, and the nurse visited her; the bowels had not been moved, and an
enema was given. On November 3 rd temperature was still up, enema was given. On November 3rd temperature was still up, and she was admitted to Sparkhill at 7 p.m., with a temperature
of $102.6^{\circ}$; pulse, 132 . Two intrauterine swabs were taken and of $102.6^{\circ}$; pulse, 132 . Two intrauterine swabs were taken and vaccine was prepared. For two days after admission her temperature came down to nearly normal, but a smelling. purulent, bloody discharge persisted, so on the fourth day after admission a blunt curetting was done, and a largish piece of septic placenta was removed; her temperature rose to $102.5^{\circ}$ on the same evening. The next morning she had an injection of vaccine, 2 minims-that was on the second morning. Temperature kept up till the fourth morning, when it began to fall, and fell in twenty-four hours to normal, and kept so for two days. Sixth day, injection of vaccine, 3 minims. On the seventh dav temperature rose to $101^{\circ}$, and remained irregular, up and down tempil the thirteenth day. On the tenth day, up to which she had looked and felt well, she complained of pain in the left side of the chest; on examination of her chest I could find slight impairthe chest; on examination of her chest I could find slight implair-
ment of the breath sounds at both bases, and diminished ment of the breath sounds at both bases, and diminished
movement of the left side of the chest. Pain passed off, morement of the left side of the chest. Pain passed off, complained again of pain in the left side in the lower axillary complained again of pain in the left side in the lower axillary
region and in the left shoulder, especially marked at end of inspiration. On the twenty-first day, that is, November 27th, she was examined by Dr. Mackey. Movement of duller still behind, especially at the base, where the breath sounds were very weak, but breathing not bronchial, though expiration heard better than inspiration. Vocal resonance resembles aegophony, and there is whispering pectoriloquy. No pleural fiction heard. Right side normal. On November 28th, or twenty-second day, 6 minims of vaccine, but her tema perature remained irregular, between $99.5^{\circ}$ and $102^{\circ}$. On December 3rd, the dnllness being more marked and increasing the chest was aspirated; no fluid found. On December 7 th aspirated again, and found fluid, and drew off 2 pints of strawcoloured, slightly thickened fluid, after which, from the 6th to
the 16th, her temperature was still up at night and down in the the 16th, her temperature was still up at night and down in the morning, varying from normal to $100^{\circ}$ and $100.5^{\circ}$. The aspirator was used again on the 16th, and between 3 and 4 oz. more of
fluid were drawn off. The fluid contained chains of streptococci similar to those of the uterus. Injections of vaccine on December 2nd, 7 th, 12th, and 19th were given, increasing the dose by 1 minim each time, but with no effect on the temperature until after the second aspiration, after which her temperature rose twice, on the 18 th and 19th, to over $100^{\circ}$, but afterwards remained normal till December 31st, when she went home feeling quite well, and has remained so. On December 30th Dr. Mackey reported left chest somewhat dull, air entry poor, but breath sounds better heard than formerly, and vocal resonance good. Aspiration in two places, no fluid. The patient all along looked fairly well in spite of her high temperature and quick pulse and effusion into the pleura.

\section{CASE $\mathrm{x}$.}

Mrs. D., aged 31. Eighth pregnancy. Admitted January 21st 1912, to the Maternity Hospital, and confined in the evening of the same day. Before admission she was examined by a midwife and by a doctor. Presentation transverse. On the 20 th, at 1 n.m. the membranes ruptured, but no pain; at 11 p.m. a limb presented, and the doctor who was called in sent her to the maternitiy hospital. She was admitted at 4 a.m. on the 21st. Examination on admission showed an arm presenting; there were no pains, and at 6 o'clock in the morning Dr. Potts did internal version, bringing down a leg. The patient had no more pain till 5 p.m., when sharp, rapid pains came on and the baby was quickly born; placenta delivered in half an hour (manually). An intrauterine douche was given. At the end of labour the pulse was 86. There was no tear of the perineum.

January 22nd. Pulse 96; temperature $100.5^{\circ}$ F. Patient complained of pain in the right wrist. Vaginal douche twice a day.

On the next day the temperature was higher-over $101^{\circ}$; and the next day it went up to $103^{\circ}$, and she complained of great pain in the right wrist and both legs. No swelling on the joints, which appeared freely movable. Very wheezy in the chest, harsh breath sounds all over the lungs; lochia offensive and scanty. The next day temperature $102^{\circ}$. An intrauterine Thomas Wilson curetted her. A great deal of clot and débris came away. The same night 20 c.cm. polyvalent antistreptococcic sarum given in the abdominal wall. The next day the patient seemed a little better, but still had pain in the wrist and the legs. The gauze packing of the curetting was remored and a douche given. Cultures grown showed streptococci.

Two days later, on January $26 \mathrm{th}$, temperature still up to $102^{\circ}$, lochia still offensive, two rigors in the morning; uterus flushed with 5 per cent. hydrogen peroxide.

January 27th. Much the same, cough more tronblesome, at left base behind the percussion note is impaired, few crepitatious and a pleuritic rub can be heard sometimes. 
January 28th. Condition the same, abdomen rather distended but no tenderness, uterine discharge clear, sputum shows pneumococcus. The patient had two rigors yesterday.

January 30th. The patient still holding her own, moist crepitations; pleural friction over the left base. Slight rigor this morning.

January 31st. The patient's aspect not so good, pulse becoming more rapid, abdominal distension increasing.

February lst. Still a good deal of distension, tenderness in the pelvis, sputum bloodstained, and a tender spot over the left buttock.

February 4th. The patient had a rigor yesterday aftermoon, temperature $104.8^{\circ}$, still feeling the effect of it to-day.

February 5th. Condition much the same, left base still dull, breath sounds deficient, abdomen not tender, and the spot on the left buttock less so.

February 6th. An abscess in the left buttock was opened, and $4 \mathrm{oz}$. of thick pus let out. Cultures of pus showed streptococci and Bacillus coli. Left base still dull.

Patient continued about the same, and examination on February 12th showed that the uterus was involuted and no hardness or tenderness in either fornix, breath sounds rather deficient in the left base, nothing else abnormal.

During the next week she had several rigors, and on February During the next week she had several rigors, and on February
19th she complained of pains in the right leg below the knee, 19th she complained of pains in the right leg below the

leg tender to the touch, and looking larger than the other. All this time her temperature was going up and down between $103^{\circ}$ and normal.

February 27th. The patient was given Burney Yeo's inhaler with creosote inhalation.

February 28th. Half grain collargol injected into basilic vein ; left base behind still dull, breath sounds deficient.

February 29th. Condition much improved, temperature having been practically below $100^{\circ}$ for three days.

From now till her discharge a month later, on March 28th, the collargol was systematically administered. The patient progressed almost imperceptibly at first, but afterwards steadily and rapidly, and when she left to fo to , but afterwards steadily sine was able to walk, and the wound in the back had quite liealed.

CASE XI.

Mrs. C., aged 26. First confinement. Patient generally septic. Confined on April 16th, 1911; attended by a midwife, who, as the labour proved difficult, called in Dr. Norton, and with great difficulty she was delivered with forceps. She remained for six days quite well, but on the seventh day she felt ill, hot, and feverish, and on the eighth day she had a rigor and remained in a condition of fever, with several rigors occurring, until admitted on April $28 \mathrm{th}$ - that is, the thirteenth day after confinement.

On admission temperature was $103^{\circ}$, abdomen tender, uterus tender with some thickening around it. Patient was nursed, bowels were kept open, and her temperature coming down gradually the first few days I hoped that the induration around the uterus and the cellulitis would absorb of itself, but in the next six days her temperature went gradually up again to $103.5^{\circ}$. I operated on May 9th, making an incision into the cellulitis I operated on May 9th, making an incision into the cellulitis
leposit per vaginam, opening two small collections of pus and deposit per vaginam, opening two small collections of pus and
draining them. The uterus was explored, and swabs taken for draining them. The uterus was explored, and swabs taken for culture and possibly vaccine preparation. For the next six and a half weeks her temperature was up every evening and down
every morning up to anything between $99.5^{\circ}$ and $102^{\circ}$ and down every morning up to anything between $99.5^{\circ}$ and $102^{\circ}$ and down
in the morning to normal, sometimes subnormal. The cultures showed pure streptococcus. She had vaccines every fourth day, but that seemed to have no effect upon the fever. The patient's general condition improved a little at first and then remained stationary; all this time she expressed herself as feeling well and free from pain though so weak that she could not sit up in bed. In the seventh week, the rise of temperature at night becoming higher, I made more frequent examinations, and on the forty-sixth day I opened another small abscess behind the uterus. The temperature fell after this, but there was still some evening rise; but gradually she became nearer and nearer to normal, and in another three weeks she was put and nearer to normal, and in another three weeks she was put to sleep out of doors in the garden. For seven more weeks she lay there looking better, feeling better, her temperature up to still that it was impossible for her to get up, and in spite of her still that it was impossible for her to get up, and in spite of her
feeling and looking better there-was no gain in weight, she feeling and looking better there-was no gain in weight, she middle of August her temperature rose again three or four times in a week to over $101^{\circ}$, and as I was away on my holiday Mr. Hewetson saw her for me, and found some induration in the right groin. He opened this, letting out some pus, on August 24th. Her temperature at once became normal, and remained

Her recovery after this was steady, but extremely slow, and even when she left the hospital she was a thin, pale, anxiouslooking woman. I saw her a month ago locking and feeling very well. Duration of stay in hospital, five months.

CASE XII.

Mrs. L., aged 32, six children. Prematurely confined on February 16th, 1911, attended by a neighbour. On the thir day was taken ill with shivers, and, getting worse, Dr. Petrie was sent for on February 22nd, and I saw her with him on February 23 rd.
Patient was wandering, and in a state of acute sepsis;-tem perature, $103.3^{\circ}$; sordes on the lips; dry, furred tongue; abdomen tender, but not distended; pulse over 140 . At the time I did not expect her to live twenty-four hours, and hesitated about moving her to the hospital for fear that she should die on the way. She had two more rigors in the first twenty-four hours after admission, and an injection of antistreptococcio serum was given, while intrauterine swabs were taken for the serum was given, while intrauterine swabs were taken for the
purpose of examination and preparation of vaccine. On the purpose of examination and preparation of vaccine. On the ( 30 million), and subsequently she had an injection of vaccine ( 30 million), and subsequently she had an injection of vaccine igors persisted and her temperature rose most days to $104^{\circ}$ or $105^{\circ}$, and sometimes to $106^{\circ}$, the vaccine treatment was stopped. The cultures made from sivabs showed pure streptococci. In the third week she had a rigor every day, her temperature on two occasions going off the chart up to $108.4^{\circ}$. On two other occasions it was up to $106^{\circ}$ and $108^{\circ}$ respectively. After the vaccine treatment was suspended blood cultures were made. and proved to be sterile. The patient was never completely conscious and her never completely conscious, and her death was expected every day, but in the ourth week she improved a very little; she only had three rigors, and for two and a half days her temperature was normal. whole higher, rising on four occasions above $102^{\circ}$ and the whole higher, rising on four occasions above $102^{\circ}$, and the
patient became more and more prostrate. At the end of the patient became more and more prostrate. At the end of the sixth week she began to cough, and her breathing was more
laboured. An examination of the chest revealed no definite laboured. An examination of the chest revealed no definite
pneumonia, no definite area of dullness, moist breath sounds. pheumonia, no definite area of dullness, moist breath sounds. patient gradually got weaker and weaker, and died on April 7th.

Post-mortem Examination.-Body well developed. Very anaemic. Abdomen: No peritonitis, though there are some shaggy tags on the caecum and lower end of ileum, as if there had long ago been some local peritonitis; uterus, etc., free and apparently normal; no pelvic cellulitis nor phlebitis. In the mushroom from the centre of the fundus covered with a yellow mushroom from the centre of the fundus covered with a yellow
purulent exudate. Liver large, normal colour, no abscesses; purulent exudate. Liver large, normal colour, no abscesses; enlarged. Thorax : No pleural effusion and no adhesions, some yellow lymph on both lungs; lungs infiltrated throughout with nodules as large as small marbles, most of these on section are abscesses and full of dirty pus, some of them are reddish and solid; evidently a septic bronchopneumonia showing infected areas in various stages; the lung between the nodules is singularly unaffected and practically normal; there is no pus in the bronchi, and none of the abscesses appear to actually communicate with a bronchus. Pericardium contains $4 \mathrm{oz}$. of serum; heart is flabby, no valvular disease.

Microscopical Examination.- The mass at the top of uterus is placenta undergoing organization, shows no evidence of acute inflammation, and contains no micro-organisms. The pus from the lung abscesses is crowded with germs of all sorts, and of types usually found in the air passages, so that although the primary lung infection was probably a metastasis, the majority smaller tubes, and the contact of the air has allowed the infection to become mixed. There are streptococci and Gram-positive tion to become mixed. There are streptococci and Gram-positive tiplococci. Gram-negative diplococci like $M$. catarrhalis and
short and long Gram-positive diphtheroid germs of all sorts.

From a consideration of these and somewhat similar cases, we learn that even when the temperature has come down to normal, the tongue has cleaned, and all abdominal pain and tenderness have disappeared, unless also the patient gains steadily in strength, puts on weight, and is able to get up, the most careful and continuous care must be taken that any surgical condition, as for example an abscess, will not be overlooked, for in these cases the abscess will form a swelling free from pain, free from tenderness, and free from redncss. We learn that on the slightest complaint of any trouble with respiration or any pain in the chest, or with any cough, a careful examination of the chest must be made. We learn that the abscess tends to form in a dependent part of the body, and I think I may add that we learn also that when these surgical conditions supervene during the time that the patient is under vaccine treatment, they are never acute, and if open to surgical treatment are easily cured.

\section{Cacsation.}

When we think of the usual surroundings of the newborn babe, we must be surprised not that there is so much puerperal fever, but that there is not a good deal more. In direct contact with the patient, in direct contact with the doctor's or midwife's hands, in direct contact with any tear or wound that may be inflicted, are, in most of the working-class houses, blankets or sheets or clothes that, cren if not describable as dirty, cannot by any stretch of the imagination be called surgically clean. It is surely legitimate to ask why sterilized towels should be placed all round the field of an operation and not around 
the field of a delivery, the latter being even more exposed to the risk of infection than the former. We take infinite pains to keep from an operation wound staphylococcus Bacillus coli, Streptococcus pyogenes, etc. Do we, can we honestly say that we do, invariably take the same pains to keep them from a cervical or perineal tear in a confinement? The doctor in attendance will invariably tell you that he thoroughly sterilized his hands, that his forceps were boiled, also any sutures and needles that he used, but is there as much attention paid to cleansing the patient as there is to cleansing the doctor's hands? I think it may truly be said that there is not, and, as I shall show you in a few minutes, it is here that the danger lies. Before a difficult forceps case is finished, the hands of the attendant must frequently come into contact with the thighs, vulva, and abdomen of the patient, and although they may be thoroughly sterilizied to start with, it is incredible that they remain so to the very end. If we hold the view that puerperal fever is only taken to a case from a previous one, that the causative germ comes into the room on the attendant's hands or instruments, and is not present on or in the patient, then the ordinary method of procedure would be sufficient; but let us examine this causative germ for a few minutes.

I give here the results of the bacteriological examination of the uterine discharge as shown in cultures or cultures and films in 21 cases of prerperal fever, nearly all of which have been under my. own care:

1. Pure Bacillus coli in cultures and films.

2. Cultures showed pure streptococcus and a few colonies of Staphylococcus auteus, sputum crowded with streptococci.

3. Streptococcus and diphtheroid bacillus.

4,5 , and 6 . Pure growth of streptococcus in cultures

7. Cultures showed streptococci and Bacillus coli mixed.

8. Profuse and pure culture of Staphylococcus albus.

8. Profuse and pure culture of Staphylococcus albus. 19. Cultures showed Staphylococcus aureus. Some doubt as to this causing puerperal rise of temperature. Three days later pus evacuated from Douglas's pouch; cultures from the pus
showed streptococcus and Bacillus coli. (This was a case of

Mr. Martin's.)

10. Cultures showed streptococcus and diphtheroid bacillus, Bacillus coli and Staphyloccus albus. The swabs were probably contaminated from the vagina. (This was a case of Dr. Edge's that died on the fourth day of peritonitis.)

2. Pure growth of streptococcus in cultures.

13. Pure growth of streptococcus in cultures, also from the pus of a secondary abscess.

14. Cultures showed profuse Bacillus coli and few streptococci.

15. Pure growth of streptococcus in cultures.

16. Pure growth of streptococcus in cultures, also from the fluid taken from the pleura.

17. A fine colony of streptococci, but not the same as the streptococci in the other puerperal cases.

18. Few. Bacillus coli, but chiefly a Gram-negative bacillus in tiny colonies.

19,20 , and 21. Pure growth of streptococcus in cultures.

Dr. Mackey, who has done all the bacteriological investigations, tells me that the streptococcus which is present in 17 of the 21 cases is quite distinct from other streptococci, is the same identically in all these 17, and that in any secondary pus, pleuritic fluid, or sputum, it was identical with that found in the uterine discharge. He also tells me that he has never found it in any other septic cases, and only once has he seen it apart from these puerperal cases, and that was in the cerebro-spinal fluid of a chorea gravidarum.

We suggest that it might be called the Streptococcus puerperalis.

Characters of Streptococcus Puerperalis.

It grows freely upon agar, producing opaque colonies which are much larger than any other streptococci; so much so, that after forty-eight hours the colonies might casily be mistaken for those. of Staphylococcus albus; in broth it produces chains of moderate length, which have little tendency to form clumps or entanglements. Its fermentation tests are as follows: It produces acid and clot in millk, aciä in lactose, in glucose, saccharose, and salicin, no change in raffinose, mannite, and inulin. These reactions show that it differs from the Streptococcus faecalis in its action to mannite, and from the Streptococcus pyogenes by its production of clot in milk. Since this definite streptococcus has been found on bacteriological examination in the uterine discharge in 17 out of 21 cases - that is, in 80 per cent. of the cases-we have a prima facie right in assuming that this is the most frequent cause of puerperal fever. It must not, at the same time, be forgotten that cases have been shown to be due to infection by Bacillus coli, Staphylococcus aureus, and other kinds of streptococci. Since this Streptococcus puerperalis is found nowhere practically but in these puerperal cases, it would be foolish to think that doctors or midwives convey the infection to the patient, it would be still more foolish to think that the infection cannot be conveyed from one patient to another. This sounds paradoxical, but it is well known to you that puerperal fever does, and will, occur in isolated cases. A doctor will for a long time be free from a case, then he gets one, and possibly after another very long interval he will get another. If one follow the other closely, if while attending the first the second were infected, it is possible that he might have conveyed the infection from one to the other, but in isolated cases where does the infection come from? A simple explanation, and one that requires, and will get, for its proof or otherwise further investigation, is that this streptococcus, like the Streptococcus faecalis, is present in the contents of the bowel, and that the puerperal woman is very susceptible to its action. Efforts will be made to discover and isolate this germ from the faeces. Its presence in the bowel will explain everything, including those cases in which, in spite of many precautions, fever occurs.

In three of the cases reported it was associated with Bacillus coli, and it is not difficult to understand. how easily the puerperal uterus becomes infected from the rectum. Even the separating bridge of the perineum may be absent at the end of a confinement, so that the passage of the streptococcus from the rectum to the uterus is actually facilitated. That perineal and rectal operations are not followed by infection by this streptococcus we may look upon as due to the fact that all such operation wounds, when there is a possibility of discharge collecting, are drained.

Now it is obvious to everyone that after the confinement is over, the cavity of the uterus, if there is the slightest fault in involution, will remain as an inert bag containing more or less blood clot and serum. Here we have a ready soil in which these streptococci can multiply, a soil, too, that is in direct contact with a surface immediately ready for absorption. This explanation then, although not fully proved, explains both the occasional'sporadic nature of the case and also the facility with which one puerperal case will infect another.

Treatment.

Preventive.-There can belittle doubt as to the lines that should be followed in order to keep patients free from puerperal fever.

It may be recalled that the Streptococcus puerperalis was associated three times with the Bacillus coli, the Bacillus coli was found alone in one case, a profuse growth of Bacillus coli with a few streptococci occurred in another, the Staphylococcus aureus in yet another, and lastly, a.fine colony of streptococci, unlike the Streptococcus puerperalis in another. Bearing these facts in mind, and also the theory that the Streptococcus puerperalis is present in the bowel, it will be recognized by all that absolute surgical cleanliness, not only of hands and of instruments, is essential, but equally essential is the absolute surgical cleanliness of the patient's skin. The whole area of the field of delivery should be thoroughly cleaned-the thighs, the vulva, and the abdomen-the hair should be clipped quite short, and if any obstetric operation has to be performed, I think it would be better to shave it off.

Since at the beginning of labour an enema is given to ensure as far as possible that the rectum sliall be empty during the delivery of the baby, it follows that the neighbourhood of the anus has recently been infected by contact with the contents of the bowel. It is our especial duty to pay greater attention than we have done to cleansing the region of the anus. However well the rectum is emptied it usually happens that more or less of the bowel contents are expelled in the last part of the second stage of labour. It is wise to have a bowl of solution of mercury biniodide $(1$ in 1,000$)$ close at hand with some biggish pieces of absorbent wool in it, and as any faecal matter escapes wipe it away thoroughly from frcnt to back with the solution; thus will any possible infection be carried away from the vaginal opening. Care should be taken not to soil one's fingers in doing so-to be successful in this the pieces of 
wool should be of large size. Always wear a sterilized gown, and take three or four sterilized towels to place under the patient and over the edges of the turned-up patient's clothes and turned down bedclothes. If there is a nurse in charge beforehand she can prepare the sterilized towels before the stage of labour at which they will be required is reached. We must cleanse our hands before we start cleansing the patient, and after cleaning the patient we must clean our own hands again, and then put on the gown and place our towels ready in position. Some of you will at this stage put on rubber gloves that have been boiled, others will not. If a man has absolute faith in the power he has of sterilizing his hands he may or may not wear gloves; if he has not this faith, then the wearing of gloves or not will make no difference; for in midwifery work above all other it frequently liappens that a hole may be torn in the glove, and unless the hand inside it is absolutely sterile the glove will have been useless.

To my mind, the great advantage of rubber gloves in surgical work is that you can by their means protect your hands from sources of infection. The method that will be used for cleansing the skin of the patient you must decide for yourselves. Personally I am a great believer in thorough scrubbing with a sterilized loofah with soap and hot water, followed by vigorous rubbing with a dry, sterilized towel, then a thorough rubbing with methylated spirits, to be followed finally by rubbing with a solution of mercury biniodide ( 1 in 500) in 75 per cent. of methylated spirits. I think I am right in saying that Mr. Leedham Green's experiments on sterilizing the hands have shown that this method gives the best results.

After what I have said, is there anything else that we can think of that will help in the prevention of puerperal fever?

I do not think it is a dream impossible of fulfilment that every woman at the commencement of labour shall have an injection of the vaccine of a Streptococcus puerperalis, thus rendering her immune beforehand to the infection by this germ. This, at any rate, would rencier her immune in 70 or 80 per cent. of the cases. Further consideration even might lead an enthusiast to inject also a vaccine prepared from the Bacillus coli.

It may be said that this elaborate method cf sterilized gowns and towels, rubber gloves, and even more, stock vaccines, will involve such an expense that the extremely moderate fee that is received by the practitioner for attending a woman at the most critical period of her life is insufficient to justify one in incurring it. Surely the occurrence of one case of puerperal fever will involve such an expenditure of brain power and of time that this very moderate expenditure in every case is to my mind not only justified hut essential. I speak now of the expenditure on the methods of sterilization, and not of the expenditure on stock vaccines. As I have already hinted, this preliminary injection of stock vaccines may be only a dream of mine which may or may not be fulfilled.

Before we leave the question of preventive treatment I may add, although it is hardly necessary to do so, the importance of impressing upon the patient beforehand the importance of a clean, wcll-aired room, of clean blankets, and clean sheets. If possible, too, the patient should have a good warm bath before labour begins, or as it begins.

Treatment of the Fever when the Temperature Rises.

When the first rise of temperature is in the first forty cight hours and is only a slight one, up to or just over $100^{\circ}$ when there is no great rise in the pulse-rate, and the patient does not feel very ill, the best thing to do is to wash out the uterus with an antiseptic solution, seeing to it care fully that all the apparatus used is scrupulously clcan. In many cases the temperature will fal the next morning and never rise again. If the rise in temperature over $100^{\circ}$ persists over two days, or occurs a second time, we must conclude that there is fever more or less severe.

It would be $\hat{a}$ very good thing if every case of puerperal fever were immediately notified to the medical officer of health, and then institutional treatment will be available for those who cannot possibly afford suitable treatment at home. The majority of these latter will probably be the midwives' cases to which, when the temperature rises, the doctor has been called in. Those who can afford suitable treatment can be immediately treated at home; but imm:ediate notification means immediate treatment for those who cannot so afford it. The number of cases at present notified to the medical officer of health in this city is only a small proportion of the cases that occur. In fact, it is actually less than the number admitted to the ward at Sparkhill.

For any treatment to have a chance of success it must be started early in the disease. What I would advise is this: That the patient be placed on her left side with the knees drawn up, that the dcctor, wearing sterilized rubber gloves to protect his own hands from infection, should pass a duck-bill speculum and swab out the vagina with a mild antiseptic solution by bits of wool held in a vulsellum forceps. Having done this he should catch hold of the anterior lip of the cervix with the vulsellum forceps, and pull the uterus a little downwards and forwards so as to expose the external os; a second person will, of course, hold the speculum, and then the doctor, holding the forceps in the right hand, will take a sterilized swab such as I show here, and, taking great care that it does not touch any of the parts on the way, will push it through the os into the interior of the uterus, will draw it out with equal care, and immediately cork it tightly in a sterilized test tube. By bacteriological examination of this swab he las a certain means of ascertaining the nature of the infection. He should then by manual examination try to satisfy himself that the interior of the utcrus is clear of any retained placenta or membranes. If therc is any doubt of this, holding the cervix as before in the vulsellum forceps, he can pass a blunt curette into the uterus and run it over the whole of the inner surface of the nterus, then swab out the interior with a piecc of wool cither on a wooden probe or held on long forceps and dipped in the dilute solution of mercury biniodide, and finally insert into the uterus a strip of iodoform gauze to act as a drain. Following on this I think it would be advisable to give the patient an injection of vaccine containing 25 or 30 million of the Streptococcus puerperalis.

I do not doubt that very shortly stock vaccines will be prepared from this streptococcus and will be placed on the market.

Believing as I do in the efficacy of a vaccine prepared from the source of the patient's infection, what I may call the patient's own vaccine, $I$ would advise that whenever the pationt can afford it, one should be prepared, and an injection of it given as soon as possible. The question of expense must enter into this treatment; the proparation of vaccine involves several hours' work, and can only be done with absolute safety either by or under the immediate supervision of a skilled bacteriologist, and such services must be paid for.

I think I may claim from some of the results in the treatment by this method that I am justified in asking for its adoption. I quoted three severe cases where thîs treatment was applied early with complete success, and even in those prolonged, drawn-out cases in which the vaccine did not appear to have an immediate curative effect. I would point out that when what I call a surgical condition, sucli as the formation of an abscess, appeared, it was only accompanied by a slight irregular temperature, by no fresh general septic condition of the patient, and after the abscess was opened the recovery was immediate and complete; whereas, in cases which did not have the vaccine treatment, any fresh surgical condition was accompanied by an increase in the general septic condition of the patient, leading ultimately to a fatal end.

I do not claim for one minute that, if the patient has been profoundly infected by the toxins of these streptococci, and if the infection has been prolonged for some time, it is possible to bring about immunity and recovery by the injection of vaccines; but what I lo ask that we should all aim at is that the patient should be given a chance of becoming immune to the infection before her powers of resistance have been too far overcome by the degree and the duration of the infection.

I would like to add here one or two other examples of treatment by this method. Let me give you a case that has come under my care after most of this lecture was written:

Mrs. J., aged 25. Confmed April 8th, normal confinement, primipara. Had a rigor April 9th, temperature $104.2^{\circ}$. Admitted to the hospital on Ap; il lith. On admission in the night I was rung up by the matron, who told me that the patient was so ill that she did not think she would live till the 
morning. I ordered stock vaccine-30 million of the Streptococcis puerperalis. When I saw her the next morning her temperature had begun to go down; she was delirious, trying to get out of bed, picking at the bediclothes, dry and almost black tongue, sordes on her lips, pulse that I could hardly count and very feeble, uterus big and tender, abdomen slightly distencled and tender, no tear in the perineum. I took swabs of the interior of the uterus. Dr. Mackey reported an infection the interior of the uterus. Dr. Mackey reported an infection her temperature fell to $101^{\circ}$, but went up again the next day to
$104.8^{\circ}$, and she had two rigors. Her temperature remained up until the third day after admission, when she had a dose of her until the third day after admission, when she had a dose of her
own vaccine, 4 minims, containing 20 million streptococci and 100 million Bacillus coli. Her temperature came down rapidly to normal, and has never risen again. Although it was some days before she became quite clear in her mind and before her tongue became clean and moist, she had a second dose of her own vaccine four days later of 25 million streptococci and 120 million Bacillus coli. Her temperature remained normal, but on May 5th I noticed a small swelling at the back of the right buttock. The next day I found that this was larger and fluctuating, and under a local anaesthetic I opened it and le out about $1 \mathrm{oz}$ of pus, there being no rise of temperature; and by this time the patient, who had had altogether four doses her own vacine, was expressing half os feeling doses of her own vaccine, was expressing herself as feeling very well certainly have said when I first saw her that this patient had not a chance of recovery.

Showing the aivantage of treatment by the patient's own vaccine as compared with stocl vaccine, there is the case of

CaSE Xiv.

Mrs. T., aged 36. Confined by a midwife ; fairly profuse postpartum haemorrhage; on second, third, and fourth days had rigors; on the fifth she was seen by Dr. Barber and by myself; temperature $103.6^{\circ}$; in acute septic condition. Admitted to the hospital the next das, and the usual treatment was followed out. Dr. Macker's report showed that infection was due to out. Dr. Mackey's report showed that infection was due to Bacillus coli, but chiefly to a Gram-negative bacillus in tiny
colonies smaller than the streptococcic colonies, which I think I am right in saying that Dr. Mackey said he had never seen before. Her temperature was irregular for the first nine davs before. Her temperature was irregular for the first nine days
after admission, but usually varying from $101^{\circ}$ to $103^{\circ}$. After the second dose of her own vaccine on the rinth day, containing 60 million Bacillus coli and 15 million of the other bacillus, her temperature came down steadily, and, although it subsequently ros z twice to 101, her recovery was continuous and complete.

CASE Xv.

A second case, also showing the advantage of her own vaccine, was that of Mrs. P. who was admitted on the fourteenth day after her confinement with all the symptoms of puerperal fever. afultures Cultures coccus aureus. On admission she had a bad cough, there were crepitations behind the left apex, and the sputum was crowded with the same streptococci. She had one dose of her own vaccine, containing 20 million streptococci and 200 million staphrlococci; her temperature came down to normal and never rose again.

In addition to this treatment by vaccines, none of the ordinary methods of treatment should, if indicated, be neglected; intrauterine douching with dilute antiseptics, iodoform pencils in the vagina if the discharge is offensive, tonics, careful attention to the bowels, and, when necessary, stimulants.

As I have said, I chose the subject because I thought it was one of interest to the majority of medical men. I may say now that I chose it also because I was anxious to impress upon you the possible advantage that your patients may derive from your knowledge of this vaccine treatment. I may bave formed my conclusions too hastily, and you may say that they are not warranted by the number of cases investigated. I can only say now that I am going on with these investigations, and further results will be published when they are ready. I chose this subject, too, because it has always seemed to me that the death of a woman in childbirth from puerperal fever is a matter of reproach to the medical profession, and any possible advantage in treatment that may be derived from investigations into all aspects of the disease should be, and will be, I am sure, heartily welcomed by the profession.

Before I close I would appeal to the Central Midwives Board to extend the duration of the course of training for midwives to at least six months. At present it is four months, and, when one thinks of what has to be learnt, this time hardly seems long enongh. I would appeal to the doctors to notify their cases immediately, so that the really poor may get institutional treatment as early as possible. I would congratulate the Faculty of Medicine herc on the near approach of the time when their students will receive part of their instruction in midwifery in a maternity hospital.
I would express my consciousness of the inadequacies of my lecture; any little merit that there may be in it I owe almost entirely to onr bacteriologist, Dr. Mackey. To him I give my deepest thanks. I have also to thank, and I do it with pleasure, my colleagues on the staffs of the Women's and Maternity Hospitals.

\section{THE ETIOLOGY OF TORSION OF THE TESTIS.}

BY R. W. MURRAY, F.R.C.S.,

SURGEON, NORTHERN HOSPITAL, LIVERPOOL.

My attention has been directed lately to torsion of the testicle, and without making by any means an exhaustive inquiry, I have endeavoured to learn what I could regarding the etiology of this remarkable complaint.

The liability of an undescended testicle to become suddenly and acutely inflamed was well known to surgeons of old, and, though often unaccounted for, was usually attributed to a blow or a strain.

The chief interest then, as now, was in distinguishing between an inflamed inguinal testicle and a piece of strangulated gut. Percival Pott mentions the case of a young man in whom inflammation of an undescended testicle caused symptoms closely rescmbling those of a hernia strangulated. The local tenderness was extreme, spread to the abdomen, and was associated with vomiting. It was noticed that the testicle on this side was not in the scrotum. Fomentations were applied and the patient recovered without an operation.

Sir Astley Cooper, in his work, The Testis, and Samuel Cooper, in his Surgical Dictionary, 1823, both draw attention to the fact that there may be considerable difficulty in distinguishing between an inflamed testicle in the inguinal canal and a strangulated knuckle of bowel. However, the older surgeons had little opportunity of studying the pathology of the living, for until well beyond the middle of the nineteenth century operative interference was confined to minor cases or to those of extreme emergency. Even when strangulation of the bowel occurred operation was invariably postponed until all other measures for obtaining relief had been tried and failed; and as thrombosis due to torsion of the cord, even when unrelieved, very rarely ends fatally, there was in those days no opportunity of determining the cause of the trouble. However, with the dawn of antiseptic surgery more light was thrown upon many obscure problems, for there was much less hesitation in making an exploratory incision. Still, many years passed before it was proved that torsion of the cord or testis was the essential cause of spontaneous inguinal orchitis. Mr. Jacobson, in his admirable article on diseases of the male organs of generation in Holmes's System of Surgery, describes the case of a lad 19 years of age, in whom inflammation of an inguinal testis produced symptoms simulating those of strangulated hernia.

The lad came to Mr. Jacobson's out-patient clinic in November, 1879, and was admitted with a view to operation. An incision over the swelling disclosed a gangrenous testicle, and an adjacent black, cyst-like mass was found to be epididymis, with above this the reddish, pulpy, oedematous cord. The cord was divided after being ligatured, and the affected parts removed.

In this case the symptoms had originated without any apparent cause, and in the light of our present knowledge there can be no doubt, though no mention was made of the fact, that it was an instance of thrombosis and gangrene following upon torsion of the cord.

So far as I have been able to ascertain, the first surgeon to describe torsion of the testicle was Professor C Nicoladini. In the Archiv fïr klinische Chirurgie, 1885, he relates the two following cases:

A man, 62 years of age, was operated upon by Professor Nicoladini in June, 1882, on account of a painful swelling of the right side of the scrotum. The swelling was of recent origin and could not be accounted for. The right side of the scrotum was considerably swollen. It was oedematous, painful, and fluctuated. It was thought suppuration had occurred. On making an incision bloodstained fluid escaped, followed by a testicle and epididymis, which were of a dark plum colour. and at first sight thought to be a piece of strangulated intestine. Further examination silowed that this extreme congestion was due to the spermatic cord being twisted like a screw was due to the spermatic cord being twisted like a screw
through more than 180 degrees, thus causing thrombosis of the 